\title{
ON AN APPROXIMATE FUNCTIONAL EQUATION OF PALEY*
}

BY

\author{
W. C. RANDELS $\dagger$
}

The purpose of this paper is to present and extend a method which has been found useful in the construction of "gegenbeispiels." The methods of proof have all been used before by various writers, but the conditions used here are more general than those considered by the other writers.

If we have a sequence of positive numbers $\left\{a_{n}\right\}$ such that

$$
\sum_{n=1}^{\infty} a_{n}=\infty
$$

and such that the function,

$$
f(z)=\sum_{n=1}^{\infty} a_{n} z^{n}
$$

is analytic inside the unit circle, we know that $f\left(\rho e^{i \theta}\right)$ will tend to infinity as $\rho \rightarrow 1$, at least for $\theta=0$. If we introduce a sequence of factors $\left\{e^{i b_{n}}\right\}\left(b_{n}\right.$ real), the function

$$
f(z)=\sum_{n=1}^{\infty} a_{n} e^{i b_{n} z^{n}}
$$

will tend to have its singularities spread over the unit circle and the order of $\max _{0 \leqq \theta<2 \pi} f\left(\rho e^{i \theta}\right)$, as $\rho \rightarrow 1$, will be decreased. This suggests the problem of determining a sequence $\left\{b_{n}\right\}$ corresponding to the sequence $\left\{a_{n}\right\}$ such that the order of $f\left(\rho e^{i \theta}\right)$ as $\rho \rightarrow 1$ is the same for all values of $\theta$.

The first result of this nature was given by Hardy and Littlewood [2]. $\$$ They considered the case of $a_{n}=n^{\beta-1 / 2}$ and they proved that

$$
f_{\beta}(z)=\frac{H t^{-\beta}}{\log a} e^{-\pi i / 4} \sum_{n=1}^{\infty} n^{\beta-1 / 2} \exp [i \alpha n \log n] z^{n}=F(\sigma)+\phi(\sigma), \S
$$

where

* Presented to the Society, February 23, 1935; received by the editors February 11, 1937.

$\dagger$ This work was done while the author was a Sterling Research Fellow at Yale University. The problem was suggested by Professor Hille.

$\ddagger$ The numbers in brackets refer to the references at the end of the paper.

$\S$ To make the printing easier we shall sometimes write $\exp [x]$ instead of $\mathrm{e}^{x}$. 


$$
\alpha=\frac{2 \pi}{\log a}, \quad H=\frac{(2 \pi)^{\beta}}{(\log a)^{\beta+1 / 2}}, \quad z=\rho e^{i \theta}, \quad \rho=e^{\alpha \sigma / t}, \quad \theta=\alpha \log \left(\frac{\alpha}{e t}\right),
$$

and

$$
\begin{aligned}
& F(\sigma)=\sum_{\nu=1}^{\infty} a^{\beta \nu} e^{-(\sigma+i t) a^{\nu},} \\
& \phi(\sigma)= \begin{cases}A+o(1), \text { as } \sigma \rightarrow 0, & \text { if } \beta<\frac{1}{2}, \\
O\left(\log \frac{1}{\sigma}\right), & \text { if } \beta=\frac{1}{2}, \\
O\left(\sigma^{-\beta+1 / 2}\right), & \text { if } \beta>\frac{1}{2} .\end{cases}
\end{aligned}
$$

The function $F(\sigma)$ is similar to the Weierstrass non-differentiable function, and extensive work by Hardy [1] on that function makes it possible to show that $f_{\beta}(z)$ is continuous for $|z|=1$ and $\beta<0$, and

$$
f_{\beta}(z)= \begin{cases}O\left\{(1-|z|)^{-\beta}\right\}, & \beta>0, \\ O\left\{\log \left(\frac{1}{1-|z|}\right)\right\}, & \beta=0 .\end{cases}
$$

Moreover they showed that*

$$
f_{\beta}(z)=\Omega\left\{(1-|z|)^{-\beta}\right\} \text { as } \rho \rightarrow 1, \quad \beta>0 .
$$

As a consequence of (1) and a theorem of Hardy and Littlewood [4, Theorem 4] it follows that

(3) $\quad \omega\left(f_{\beta}, h\right)=\underset{\left|\theta_{1}-\theta_{2}\right| \leqq h}{\text { l.u.b. }}\left|f_{\beta}\left(e^{i \theta_{1}}\right)-f_{\beta}\left(e^{i \theta_{2}}\right)\right|=\left\{\begin{array}{l}O\left(h^{-\beta}\right),-1<\beta<0, \\ O\left(h \log \frac{1}{h}\right), \quad \beta=-1 .\end{array}\right.$

Hille [5] has given a proof of (1), but by his method one cannot prove (2). Hille, and Ingham [6], considered the similar functions

$$
f_{\beta \gamma}(z)=\sum_{n=2}^{\infty} n^{-1 / 2}(\log n)^{-\gamma} e^{i n(\log n) \beta} z^{n}, \quad \beta<1, \quad \gamma>\frac{1}{2}(\beta+1),
$$

and showed that these functions are continuous for $|z|=1$.

It is natural to try to prove similar theorems for functions of the type

$$
f(z)=\sum_{n=1}^{\infty} b(n) e^{i \Delta(n)} z^{n} .
$$

It is convenient to assume that the functions $b(x)$ and $\Delta(x)$ are defined for

* By the notation $f(x)=\Omega\{g(x)\}$ we mean that $f(x) \neq o\{g(x)\}$. 
all values of $x$ on the interval $(1, \infty)$ and we shall do so from now on. This problem was first handled by Paley [7] who showed that, if $n_{\nu}(\theta)$ is defined by the relation

$$
\Delta^{\prime}\left\{n_{\nu}(\theta)\right\}=2 \pi \nu-\theta,
$$

then

$$
\begin{aligned}
f(z)= & \sum_{n=1}^{\infty} b(n) e^{i \Delta(n) z^{n}} \\
= & \pi^{1 / 2} e^{\pi i / 4} \sum_{\nu=1}^{\infty} \frac{b\left\{n_{\nu}(\theta)\right\}}{\left(\Delta^{\prime \prime}\left\{n_{\nu}(\theta)\right\}\right)^{1 / 2}}|z|^{n_{\nu}(\theta)} \exp \left[i \left\{\Delta\left[n_{\nu}(\theta)\right]+\theta n_{\nu}(\theta)\right.\right. \\
& \left.\left.-2 \pi \nu n_{\nu}(\theta)\right\}\right]+R(z),
\end{aligned}
$$

where $R(z)$ is continuous for $|z|=1$. Paley's results however were not as general as ours for he used a condition of the form

$$
b(n)=O\left(x^{\epsilon-1 / 2}\right), \quad \epsilon<1 / 10 .
$$

Wilton [8] working on the same series when $|z|=1$ proved the equation.

$$
\begin{aligned}
f_{m}\left(e^{i \theta}\right)= & \sum_{n=1}^{m} b(n) e^{i \Delta(n)} e^{i n \theta} \\
= & \pi^{1 / 2} e^{\pi i / 4} \sum_{\nu=1}^{\mu} \frac{b\left\{n_{\nu}(\theta)\right\}}{\left(\Delta^{\prime \prime}\left\{n_{\nu}(\theta)\right\}\right)^{1 / 2}} \exp \left[i\left\{\Delta\left[n_{\nu}(\theta)\right]+\theta n_{\nu}(\theta)-2 \pi n_{\nu}(\theta)\right\}\right] \\
& +O(1)+o\left(\int_{1}^{\mu} \frac{b(x)\left[\Delta^{\prime \prime}(x)\right]^{1 / 2}}{\Delta^{\prime}(x)} d x\right),
\end{aligned}
$$

where $n_{\mu}(\theta) \leqq m<n_{\mu+1}(\theta)$. Wilton mentions that his equation is in general a special case of one of van der Corput. We notice that neither the method of Paley nor that of Wilton will prove (1) or (2).

The purpose of this paper is to give an extension of Paley's methods so as to include (1) as a special case. In $\$ 1$ we prove a functional equation similar to (4) except that $R(z)$ is not necessarily continuous. The functional equation of $\S 1$ could possibly be gotten from Wilton's (5) by replacing $b(x)$ by $\rho^{x} b(x)$ but it would involve at least as many complications as the proof given here.

In $\S 2$, it is shown by a method of Hardy [1] that

$$
\begin{aligned}
G(z) & =\pi^{1 / 2} e^{\pi i / 4} \sum_{\nu=1}^{\infty} \rho^{n_{\nu}} \frac{b\left(n_{\nu}\right)}{\left[\Delta^{\prime \prime}\left(n_{\nu}\right)\right]^{1 / 2}} \exp \left[i\left\{\Delta\left(n_{\nu}\right)+\theta n_{\nu}-2 \pi \nu n_{\nu}\right\}\right] \\
& =O\left\{M\left(\frac{1}{1-\rho}\right)\right\}
\end{aligned}
$$


where, if $n_{\mu}(0) \leqq x<n_{\mu+1}(0)$,

$$
M(x)=\sum_{\nu=1}^{\mu} \max _{n_{\nu-1}(0) \leqq y \leqq n \nu(0)} \frac{b(y)}{\left[\Delta^{\prime \prime}(y)\right]^{1 / 2}}+\max _{n_{\mu}(0) \leqq y \leqq x} \frac{b(y)}{\left[\Delta^{\prime \prime}(y)\right]^{1 / 2}} .
$$

We also show that

if

$$
G(z)=\Omega\left\{M\left(\frac{1}{1-\rho}\right)\right\}
$$

$$
M(x)=O\left\{\frac{b(x)}{\left[\Delta^{\prime \prime}(x)\right]^{1 / 2}}\right\} .
$$

The remainder function $R(z)$ is studied in $\S 3$ and it is shown that if $M(x)$ is bounded, $R(z)$ is continuous for $|z|=1$ and otherwise

$$
R(z)=o\left\{M\left(\frac{1}{1-\rho}\right)\right\} .
$$

In $\$ 4$ we use a method of Hardy and Littlewood [4] to show that if $f(z)$ is analytic for $|z|<1$ and continuous for $|z|=1$, and

$$
f^{\prime}(z)=O\{g(|z|)\}
$$

where $g(x) \uparrow \infty$ as $x \rightarrow 1$, then

$$
\omega(f, h)=\underset{\left|\theta_{1}-\theta_{2}\right| \leqq h}{\operatorname{lub}}\left|f\left(e^{i \theta_{1}}\right)-f\left(e^{i \theta_{2}}\right)\right|=O\left\{\int_{1-h}^{1} g(x) d x\right\},
$$

provided the integral exists.

Wilton [8] has given some applications of his equation so that in discussing applications we confine ourselves to cases which have not been covered by Wilton. It is pointed out that (1), (2), and (3) follow from this theory and also certain generalizations of them are possible. Ingham's results also follow as pointed out by Wilton, and furthermore

$$
\omega\left(f_{\beta \gamma}, h\right)=O\left\{\left(\log \frac{1}{h}\right)^{-\gamma-\beta / 2+3 / 2}\right\}, \quad \text { if } \gamma+\frac{\beta}{2}>\frac{3}{2},
$$

where $f_{\beta \gamma}(z)$ is defined by

$$
f_{\beta \gamma}(z)=\sum_{n=2}^{\infty} n^{-1 / 2}(\log n)^{-\gamma} \exp \left[i \int_{1}^{n}(\log x)^{\beta} d x\right] .
$$

It is finally shown that, if $b(x)$ satisfies conditions (A), (B), and (C) of $\$ 1$, and 


$$
\int_{1}^{x} b^{2}(x) d x=O\left\{x^{1+\epsilon} b^{2}(x)\right\}, \quad \epsilon<1 / 30
$$

and

$$
\int_{1}^{\infty} b^{2}(x) d x=\infty
$$

then, if $\Delta(x)$ is defined by

$$
\Delta(x)=\int_{2}^{x} \log \left[\int_{1}^{y} b^{2}(z) d z\right] d y,
$$

the functions $\Delta(x)$ and $b(x)$ will satisfy condition (D) of $\$ 2$ which implies that the singularities of the function

$$
f(z)=\sum_{n=2}^{\infty} b(n) e^{i \Delta(n)} z^{n}
$$

are distributed uniformly over the unit circle, and the order of $f\left(\rho e^{i \theta}\right)$ as $\rho \rightarrow 1$ is the same for all values of $\theta$. This answers a question proposed in the opening paragraph.

1. The functional equation. The method used in this section is essentially that of Paley and the notation is chosen to agree with his. If the functions $b(x)$ and $d(x)$ are given we define

$$
\begin{aligned}
\Delta(x) & =\int_{1}^{x} d(y) d y, \\
z & =\rho e^{i \theta}, \\
d\left\{n_{\nu}(\theta)\right\} & =2 \pi \nu-\theta, \quad 0 \leqq \theta<2 \pi, \quad \nu=1,2, \cdots, \\
F(\nu ; \theta) & =\Delta\left\{n_{\nu}(\theta)\right\}+\theta n_{\nu}(\theta)-2 \pi \nu n_{\nu}(\theta) .
\end{aligned}
$$

We impose the following conditions on $b(x)$ and $d(x):^{*}$

(A) The function $d(x)$ has two derivatives, continuous on the open interval $(1, \infty)$ and

$$
\begin{array}{rlrl}
d(x) & \uparrow \infty \text { as } x \rightarrow \infty, \\
d^{\prime}(x) & =O\left(x^{-1}\right), & \\
1 / d^{\prime}(x) & =O\left(x^{1+\epsilon}\right), & & 0<\epsilon<1 / 30, \\
d^{\prime \prime}(x) & =O\left(x^{\eta-2}\right), & & \eta<1 / 10-\epsilon .
\end{array}
$$

We notice that the first condition on $d(x)$ implies that $n_{\nu}(\theta) \uparrow \infty$ as $\nu \rightarrow \infty$.

${ }^{*}$ It would obviously be sufficient to assume that these conditions are satisfied only for $x$ greater than a certain $x_{0}$; but to simplify the work we carry through the proof for $x_{0}=1$. 
(B) The function $b(x)$ is monotone and has one derivative continuous on $(1, \infty)$ and

$$
b^{\prime}(x)=O\left\{b(x) x^{\delta-1}\right\}, \quad \delta \leqq \epsilon .
$$

The function $d^{\prime}(x)$ and $b(x)$ satisfy the condition:

(C) There exists a constant $k>1$, such that for each function

$$
f\left(x^{\prime}\right)<k f(x), \quad k f\left(x^{\prime}\right)>f(x), \quad \text { if } \quad 1 \leqq x \leqq x^{\prime} \leqq e x .
$$

If we had restricted ourselves to functions of the logarithmic-exponential type, condition (C) would mean that the functions could not be like $e^{x}$ or $e^{-x}$. We notice some consequences of (C). If $f(x)$ satisfies (C), then

$$
\frac{1}{f\left(x^{\prime}\right)}<\frac{k}{f(x)}, \quad \frac{k}{f\left(x^{\prime}\right)}>\frac{1}{f(x)}, \quad 1 \leqq x \leqq x^{\prime} \leqq e x .
$$

If two functions $f_{1}(x)$ and $f_{2}(x)$ satisfy (C) with constants $k_{1}$ and $k_{2}$ respectively, then for $x \leqq x^{\prime} \leqq e x$,

$$
f_{1}\left(x^{\prime}\right) f_{2}\left(x^{\prime}\right)<k_{1} k_{2} f_{1}(x) f_{2}(x), \quad k_{1} k_{2} f_{1}\left(x^{\prime}\right) f_{2}\left(x^{\prime}\right)>f_{1}(x) f_{2}(x) .
$$

If $f(x)$ satisfies (C), $f(x)>0$ then for $x \leqq x^{\prime} \leqq e x$,

$$
k \int_{x^{\prime}}^{A} f(y) d y>\int_{x}^{A+x-x^{\prime}} f(y) d y, \quad k \int_{x^{\prime}}^{\infty} f(y) d y>\int_{x}^{\infty} f(y) d y,
$$

and

$$
\begin{aligned}
& \int_{0}^{x^{\prime}} f(y) d y<e k \int_{1}^{x} f(y) d y, \\
& \int_{1}^{x^{\prime}} f(y) d y<e k \int_{1}^{x} f(y) d y+\int_{1}^{e} f(y) d y .
\end{aligned}
$$

This means that, if the functions $f_{1}(x)$ and $f_{2}(x)$ satisfy (C) and are positive, then the functions

$$
\frac{1}{f_{1}(x)}, \quad f_{1}(x) f_{2}(x), \quad \int_{x}^{\infty} f_{1}(y) d y, \quad \int_{1}^{x} f_{1}(y) d y
$$

also satisfy (C) for $x>a>1$ for some $a$. If $f(x)>0$ satisfies (C), then for $e^{n} x \leqq x^{\prime}<e^{n+1} x$,

$$
\frac{f\left(x^{\prime}\right)}{f(x)}=\frac{f(e x)}{f(x)} \cdots \frac{f\left(x^{\prime}\right)}{f\left(e^{n} x\right)}<k^{n+1}<k \cdot k^{\log \left(x^{\prime} / x\right)}=k\left(\frac{x^{\prime}}{x}\right)^{\log k} .
$$

If $f(x)>0$ satisfies (C), then 


$$
\sum_{n=1}^{\infty} f(n)<k \sum_{n=1}^{\infty} \int_{n}^{n+1} f(x) d x=k \int_{1}^{\infty} f(x) d x
$$

and

$$
\int_{1}^{\infty} f(x) d x=\sum_{n=1}^{\infty} \int_{n}^{n+1} f(x) d x<k \sum_{n=1}^{\infty} f(n),
$$

so that the series and integrals are equiconvergent.

We wish to obtain an approximate functional equation for the function,

$$
f(z)=\sum_{n=1}^{\infty} b(n) \exp [i \Delta(n)] z^{n} .
$$

We first consider the group of terms*

$$
\sum_{r \rightarrow s}^{r+s} b(n) \exp [i \Delta(n)] z^{n}=\sum_{r=s}^{r+s} c(n) \exp [i \Delta(n)+i n \theta],
$$

where $r=n_{\nu}, s=n_{\nu}{ }^{3 / 5}$, and where $c(x)=b(x) \rho^{x}$. We have

$$
\begin{aligned}
\sum_{r \rightarrow s}^{r+s} c(n) & \exp [i \Delta(n)+i n \theta]-c\left(n_{v}\right) \sum_{r-s}^{r+s} \exp [i \Delta(n)+i n \theta] \\
= & O\left\{\sum_{-s}^{s}\left|c\left(n+n_{\nu}\right)-c(n)\right|\right\} \\
= & O\left\{\max _{\left|x-n_{\nu}\right| \leqq s}\left|\frac{d}{d x} c(x)\right| \sum_{0}^{s} n\right\} \\
= & O\left\{(1-\rho) b\left(n_{\nu}\right) \rho^{n_{\nu} / 2} n_{\nu}^{6 / 5}+n_{\nu}^{1 / 6+8} b\left(n_{\nu}\right) \rho^{n_{\nu} / 2}\right\},
\end{aligned}
$$

since by (C) and (B),

$$
\begin{array}{r}
c^{\prime}(x)=b(x) \rho^{x} \log \rho+b^{\prime}(x) \rho^{x}=O\left\{b\left(n_{\nu}\right) \rho^{n_{\nu} / 2}(1-\rho)+n_{\nu}^{8-1} b\left(n_{\nu}\right) \rho^{n_{\nu} / 2}\right\}, \\
\frac{1}{2} n_{\nu} \leqq x \leqq \frac{8}{2} n_{\nu} .
\end{array}
$$

By (A)

$$
\begin{aligned}
\sum_{r-s}^{r+s} \exp [i \Delta(n)+i n \theta]-\int_{r-s}^{r+s} \exp [i \Delta(x)+i x \theta-2 \pi i \nu x] d x \\
=O\left\{n_{\nu}^{3 / 5} \max _{\left|x-n_{\nu}\right| \leqq s}\left|\frac{d}{d x}[i \Delta(x)+i x \theta-2 \pi i \nu x]\right|\right\} \\
=O\left\{n_{\nu}^{3 / 5} \max _{\left|x-n_{\nu}\right| \leqq s}\left|\int_{n_{\nu}}^{x} d^{\prime}(y) d y\right|\right\}
\end{aligned}
$$

* By the symbol $\sum_{\mathrm{a}}^{b}$ we shall understand the sum over all values of $n$ such that $a \leqq n<b$. 
1938]

$$
=O\left\{n_{\nu}^{6 / 5} \max _{\left|x-n_{\nu}\right| \leqq s} d^{\prime}(x)\right\}=O\left\{n_{\nu}^{1 / 5}\right\} .
$$

We have, again using (A),

$$
\Delta(x)+x \theta-2 \pi \nu x=F(\nu ; \theta)+\left(x-n_{\nu}\right)^{2} d^{\prime}\left(n_{\nu}\right)+o\left\{\frac{\left|x-n_{\nu}\right|^{8}}{n_{\nu}^{2-\eta}}\right\},
$$

so that

$$
\begin{gathered}
\int_{r-s}^{r+s} \exp [i \Delta(x)+i x \theta-2 \pi i \nu x] d x-\exp [i F(\nu ; \theta)] \int_{r-s}^{r+s} \exp \left[i\left(x-n_{\nu}\right)^{2} d^{\prime}\left(n_{\nu}\right)\right] d x \\
=O\left\{\int_{-s}^{s}\left|1-\exp \left[i O\left(\frac{x^{3}}{n_{\nu}^{2-\eta}}\right)\right]\right| d x\right\} \\
=O\left\{\frac{1}{n_{\nu}^{2-\eta}} \int_{-s}^{s}\left|x^{3}\right| d x\right\}=O\left\{n_{\nu}^{2 / 5+\eta}\right\} .
\end{gathered}
$$

By a simple change of variable

$$
\int_{r-s}^{r+s} \exp \left[i\left(x-n_{v}\right)^{2} d^{\prime}\left(n_{v}\right)\right] d x=2\left[d^{\prime}\left(n_{v}\right)\right]^{-1 / 2} \int_{0}^{t} e^{2 x^{2}} d x,
$$

where $t=n_{\nu}^{3 / 5}\left[d^{\prime}\left(n_{v}\right)\right]^{-1 / 2}$, and we know that

$$
\int_{0}^{\infty} e^{i x^{2}} d x=\frac{1}{2} e^{\pi i / 4} \pi^{1 / 2}
$$

By considering the graph of the function $\cos x^{2}$ we see that $\int_{a}^{\infty} \cos x^{2} d x$ can be represented as an alternating series for which the absolute value of the terms is steadily decreasing. Therefore

$$
\int_{t}^{\infty} \cos x^{2} d x=O\left\{\left[d^{\prime}\left(n_{v}\right)\right]^{1 / 2} n_{\nu}^{-8 / 5}\right\} .
$$

By applying similar considerations to $\sin x^{2}$ we see that

$$
\int_{t}^{\infty} e^{i x^{2}} d x=O\left\{\left[d^{\prime}\left(n_{v}\right)\right]^{1 / 2} n_{v}^{-8 / 5}\right\}
$$

Hence

$$
\begin{aligned}
& \int_{r-s}^{r+s} \exp [i \Delta(x)+i x \theta-2 \pi i \nu x] d x- \pi^{1 / 2} e^{\pi i / 4}\left[d^{\prime}\left(n_{\nu}\right)\right]^{-1 / 2} \\
&=O\left\{n_{\nu}^{2 / 5+\eta}+n_{\nu}^{-3 / 5}\right\}=O\left\{n_{\nu}^{2 / 5+\eta}\right\}
\end{aligned}
$$


This implies that

$$
\begin{gathered}
\sum_{r \rightarrow s}^{r+s} c(n) \exp [i \Delta(n)+i n \theta]=\pi^{1 / 2} e^{\pi i / 4}\left[d^{\prime}\left(n_{\nu}\right)\right]^{-1 / 2} \exp [i F(\nu ; \theta)] b\left(n_{\nu}\right) \rho^{n_{\nu}} \\
+O\left\{(1-\rho) b\left(n_{\nu}\right) n_{\nu}^{6 / 5} \rho^{n_{\nu} / 2}+b\left(n_{\nu}\right) n_{\nu}^{1 / 5+\delta} \rho^{n_{\nu} / 2}+b\left(n_{\nu}\right) n_{\nu}^{2 / 5+\eta} \rho^{n_{\nu}}\right\} .
\end{gathered}
$$

We set $a(x)=\Delta(x)+\theta x, \gamma(x)=a(x+1)-a(x)$. We have then

$$
\sum_{r+s}^{r_{1}-s_{1}} b(n) e^{i a(n)} \rho^{n}=\sum_{r+s}^{r_{1}-s_{1}} \frac{c(n)}{e^{i \gamma(n)}-1}\left\{e^{i \gamma(n)}-1\right\} e^{i a(n)},
$$

where $r_{1}=n_{\nu+1}, s_{1}=n_{\nu+1}^{3 / 5}$, and if we define

$$
H(x)=\sum_{1}^{x}\left\{e^{i \gamma(n)}-1\right\} e^{i a(n)}=e^{i a([x+1])}-e^{i a(1)}=O(1),
$$

this gives us

$$
\sum_{r+8}^{r_{1}-s_{1}} b(n) e^{i a(n)} \rho^{n}=\int_{r+8}^{r_{1}-s_{1}} \frac{c(x)}{e^{i \gamma(x)}-1} d H(x) .
$$

Integrating by parts we have

$$
\begin{aligned}
\int_{r+s}^{r_{1}-s_{1}} \frac{c(x)}{e^{i \gamma(x)}-1} d H(x)=\left.\frac{c(x) H(x)}{e^{i \gamma(x)}-1}\right|_{r+s} ^{r_{1}-s_{1}} & \\
& -\int_{r+s}^{r_{1}-s_{1}} H(x) \frac{d}{d x}\left\{\frac{c(x)}{e^{i \gamma(x)}-1}\right\} d x .
\end{aligned}
$$

By the definition of $\gamma(x)$ and property (A),

$$
\gamma(x)=d(x)+O\left\{d^{\prime}(x)\right\}+\theta, \quad \gamma^{\prime}(x)=d^{\prime}(x)+O\left\{d^{\prime \prime}(x)\right\}
$$

and

$$
\gamma\left(n_{v}\right)-2 \pi \nu+\theta=O\left\{d^{\prime}\left(n_{v}\right)\right\} .
$$

Consequently by (C) there exists a constant $M>0$ so that for $\nu$ sufficiently large

$$
\begin{aligned}
\gamma\left(n_{\nu}+n_{\nu}^{3 / 5}\right) & -2 \pi \nu+\theta=\gamma\left(n_{\nu}\right)-2 \pi \nu+\theta+\int_{n_{\nu}}^{r+s} \gamma^{\prime}(x) d x \\
& >n_{\nu}^{3 / 5} \min _{0 \leqq x \leqq}\left[d^{\prime}\left(n_{\nu}+x\right)\right]-O\left\{d^{\prime}\left(n_{\nu}\right)+n_{\nu}^{3 / 5} d^{\prime \prime}\left(n_{\nu}\right)\right\} \\
& >M n_{\nu}^{3 / 5}\left(n_{\nu}+n_{\nu}^{3 / 5}\right)^{-1-\epsilon}
\end{aligned}
$$

and by (A) for $x>n_{\nu}+n_{\nu}{ }^{3 / 5}$, there exists an $M^{\prime}$ such that 


$$
\begin{aligned}
\gamma(x)-2 \pi \nu+\theta & >M n_{\nu}^{3 / 5}\left(n_{\nu}+n_{\nu}^{3 / 5}\right)^{-1-\epsilon}+\left(x-n_{\nu}-n_{\nu}^{3 / 5}\right) \min _{r+s \leqq y \leqq x}\left[d^{\prime}(y)\right] \\
& >M^{\prime}\left(x-n_{\nu}\right) x^{-1-\epsilon} .
\end{aligned}
$$

If we define $N_{\nu}$ by the relation

$$
d\left(N_{\nu}\right)=(2 \nu+1) \pi-\theta,
$$

then since $\gamma^{\prime}(x)=d(x+1)-d(x)>0$, we have for $x<N_{\nu}$,

$$
|\gamma(x)-2(\nu+1) \pi+\theta| \geqq \pi .
$$

We know however that, for $2 \pi \nu<y \leqq(2 \nu+1) \pi$,

$$
\left(e^{i y}-1\right)^{-1}=O\left\{(y-2 \pi \nu)^{-1}\right\},
$$

and therefore

$$
\left(e^{i \gamma(x)}-1\right)^{-1}=O\left\{x^{1+\epsilon}\left(x-n_{v}\right)^{-1}\right\}, \quad n_{\nu}+n_{\nu}^{3 / 5} \leqq x \leqq N_{\nu} .
$$

By a similar reasoning

$$
\left(e^{i \gamma(x)}-1\right)^{-1}=O\left\{n_{v+1}^{1+c}\left(n_{\nu+1}-x\right)^{-1}\right\}, \quad N_{\nu} \leqq x \leqq n_{\nu+1}-n_{v+1}^{3 / 5},
$$

and

$$
\begin{aligned}
\left(e^{i \gamma(x)}-1\right)^{-1}=O\left\{\left(\frac{n_{v+1}}{2^{j}}\right)^{1+\bullet}\left(\frac{n_{\nu+1}}{2^{j}}-x\right)^{-1}\right\}, \quad N_{\nu} \leqq & x \leqq \frac{n_{\nu+1}}{2^{j}} \\
j & =1,2, \cdots
\end{aligned}
$$

Therefore, by (C),

$$
\left.\frac{c(x) H(x)}{e^{i \gamma(x)}-1}\right|_{r+8} ^{r_{1}-s_{1}}=O\left\{c\left(n_{v}\right) n_{v}^{2 / 5+\epsilon}+c\left(\frac{1}{2} n_{v+1}\right) n_{v+1}^{2 / 5+\bullet}\right\} .
$$

We also have*

$$
\begin{aligned}
\mid \int_{r+8}^{r_{1}-s_{1}} H(x) & \frac{d}{d x}\left\{\frac{c(x)}{e^{i \gamma(x)}-1}\right\} d x \mid \\
& \leqq 2\left[\int_{r+8}^{2 n_{\nu}}+\int_{2 n_{\nu}}^{N_{\nu}}+\int_{N_{\nu}}^{n_{\nu+1} / 2}+\int_{n_{\nu+1} / 2}^{r_{1}-s_{1}}\right]\left|\frac{d}{d x}\left\{\frac{c(x)}{e^{i \gamma(x)}-1}\right\}\right| d x \\
& =2\left(I_{1}+I_{2}+I_{3}+I_{4}\right) .
\end{aligned}
$$

Using (1.5) and the fact that $\gamma^{\prime}(x)=O(1 / x)$ we have

* In case $2 n_{\nu}>N_{\nu}$ we drop $I_{2}$, if $N_{\nu}>\frac{1}{2} n_{\nu+1}$, we drop $I_{3}$, and if $\frac{1}{2} n_{\nu+1}>n_{\nu+1}-n_{\nu+1}^{3 / 5}$, we drop $I_{3}$. It is essential that $n_{\nu}+n_{\nu}^{3 / 5} \leqq n_{\nu+1}-n_{\nu+1}^{3 / 5}$. This will be shown later (cf. (2.1)). 


$$
\begin{aligned}
I_{1} & =O\left\{\int_{r+s}^{2 n_{\nu}} \frac{x^{1+\epsilon}\left|c^{\prime}(x)\right|}{x-n_{\nu}} d x+\int_{r+s}^{2 n_{\nu}} \frac{c(x) x^{1+2 \epsilon}}{\left(x-n_{v}\right)^{2}} d x\right\} \\
& =O\left\{I_{1}^{\prime}+I_{1}^{\prime \prime}\right\}
\end{aligned}
$$

and by (B) and (C)

$$
\begin{aligned}
I_{1}^{\prime}= & O\left\{\int _ { 0 } ^ { n _ { \nu } } \left[(1-\rho) b\left(n_{\nu}+x\right)\left(n_{\nu}+x\right)^{1+\epsilon_{\rho} x+n_{\nu}}\right.\right. \\
& \left.\left.+b\left(x+n_{\nu}\right)\left(x+n_{\nu}\right)^{\delta+e_{p} x+n_{\nu}}\right] \frac{d x}{x}\right\} \\
= & O\left\{\left[(1-\rho) b\left(n_{\nu}\right) n_{\nu}^{1+\epsilon} \rho^{n \nu}+b\left(n_{\nu}\right) n_{\nu}^{\delta+e} \rho^{n \nu}\right] \log n_{\nu}\right\}
\end{aligned}
$$

and

(1.10) $I_{1}^{\prime \prime}=O\left\{\int_{0}^{n_{\nu}} c\left(n_{\nu}+x\right)\left(n_{\nu}+x\right)^{1+2 \epsilon} x^{-2} d x\right\}=O\left\{c\left(n_{\nu}\right) n_{\nu}^{2 / 5+2 \epsilon}\right\}$.

Similarly

$$
I_{2}=O\left(I_{2}^{\prime}+I_{2}^{\prime \prime}\right),
$$

where

$$
\begin{aligned}
I_{2}^{\prime} & =\int_{2 n_{v}}^{N_{v}} x^{\epsilon}\left[(1-\rho) b(x) \rho^{x}+x^{\delta-1} b(x) \rho^{x}\right] d x \\
& =O\left\{\sum_{2 n_{v}}^{N_{v}} n^{\epsilon}\left[(1-\rho) b(n) \rho^{n}+n^{\delta-1} b(n) \rho^{n}\right]\right\}
\end{aligned}
$$

and

(1.12) $I_{2}^{\prime \prime}=O\left\{\sum_{2 n_{\nu}}^{N_{v}} c(n) n^{2 \bullet-1}\right\}$.

Also $I_{3}=O\left(I_{3}^{\prime}+I_{3}^{\prime \prime}\right)$, and by (1.7), if $x \geqq N_{\nu}, 2^{-j-2} n_{\nu+1} \leqq x \leqq 2^{-j-1} n_{\nu+1}$,

$$
\left(e^{i \gamma(x)}-1\right)^{-1}=O\left\{\left(n_{v+1} 2^{-j}\right)^{1+e}\left(n_{v+1} 2^{-j}-x\right)^{-1}\right\}=O\left\{x^{e}\right\}
$$

uniformly for $j=0,1, \cdots$, so that

(1.13) $I_{3}^{\prime}=O\left\{\sum_{N_{\nu}}^{n_{\nu+1} / 2} n^{e}\left[(1-\rho) b(n) \rho^{n}+n^{\delta-1} b(\dot{n}) \rho^{n}\right]\right\}$,

(1.14) $I_{3}^{\prime \prime}=O\left\{\sum_{N_{\nu}}^{n_{\nu+1} / 2} n^{2 e-1} b(n) \rho^{n}\right\}$.

Finally $I_{4}=O\left(I_{4}^{\prime}+I_{4}^{\prime \prime}\right)$ and by (1.6) 


$$
\begin{aligned}
I_{4}^{\prime} & =\int_{n_{\nu+1} / 2}^{r_{1}-s_{1}}\left[(1-\rho) b(x) n_{\nu+1}^{1+e} \rho^{x}+b(x) x^{\delta-1} n_{\nu+1}^{1+e} \rho^{x}\right]\left(n_{\nu+1}-x\right)^{-1} d x \\
& =O\left\{n_{\nu+1}^{1+e} \log n_{\nu+1}\left[(1-\rho) b\left(n_{\nu+1}\right) \rho^{n_{\nu+1} / 2}+b\left(n_{\nu+1}\right) n_{\nu+1}^{\delta-1} \rho^{n_{\nu+1} / 2}\right]\right\}, \\
(1.16) I_{4}^{\prime \prime} & =O\left\{n_{\nu+1}^{2 / 5+2 \epsilon} b\left(n_{\nu+1}\right) \rho^{n+1 / 2}\right\} .
\end{aligned}
$$

From (1.4) and (1.8)-(1.16) we get the principal result of the paper that

$$
f(z)=\sum_{n=1}^{\infty} b(n) e^{i \Delta(n)} z^{n}=G(z)+R(z),
$$

where

$$
G(z)=\pi^{1 / 2} e^{\pi i / 4} \sum_{\nu=1}^{\infty} b\left(n_{\nu}\right)\left[d^{\prime}\left(n_{\nu}\right)\right]^{-1 / 2} e^{i F(\nu ; \theta)} \rho^{n_{\nu}}
$$

and, if $\alpha=\max (\eta, 2 \epsilon)$,

$$
\begin{aligned}
R(z)= & O\left\{\sum _ { \nu = 1 } ^ { \infty } \left[(1-\rho) b\left(n_{\nu}\right) n_{\nu}^{6 / 5+\epsilon} \rho^{n_{\nu}}+b\left(n_{\nu}\right) n_{\nu}^{2 / 5+\alpha} \rho^{n_{\nu}}\right.\right. \\
& \left.+(1-\rho) b\left(n_{\nu}\right) n_{\nu}^{6 / 5+\epsilon} \rho_{\nu}^{n_{\nu} / 2}+b\left(n_{\nu}\right) n_{\nu}^{2 / 5+\alpha} \rho^{n_{\nu} / 2}\right] \\
& \left.+\sum_{n=1}^{\infty}\left[b(n) n^{2 \epsilon-1} \rho^{n}+(1-\rho) b(n) n^{\epsilon} \rho^{n}\right]\right\}=O\left\{\sum_{i=1}^{6} R_{i}(z)\right\} .
\end{aligned}
$$

We notice that this procedure is valid only for $|z|<1$. We shall now give a functional equation of the type used by Wilton (5) which is valid for $|z|=1$. The equation is

$$
\sum_{n=1}^{m} b(n) e^{i \Delta(n)} e^{i n \theta}=\pi^{1 / 2} e^{\pi i / 4} \sum_{\nu=1}^{\mu} b\left(n_{v}\right)\left[d^{\prime}\left(n_{v}\right)\right]^{-1 / 2} e^{i F(v ; \theta)}+R(m, \theta),
$$

where $n_{\mu}+n_{\mu}{ }^{3 / 5} \leqq m \leqq n_{\mu+1}+n_{\mu+1}^{3 / 5}$. It can be seen that the sums

$$
\begin{array}{ll}
\sum_{r+s}^{r_{1}+s_{1}} b(n) e^{i \Delta(n)} e^{i n \theta}, & \nu<\mu, \\
\sum_{u+v}^{m} b(n) e^{i \mu(n)} e^{i n \theta}, & m \leqq n_{\mu+1}-n_{\mu+1}^{3 / 5},
\end{array}
$$

where $u=n_{\mu}, v=n_{\mu}{ }^{3 / 5}$, can be handled by the methods which we have used and moreover

$$
\sum_{u_{1}-v_{1}}^{m} b(n) e^{i \Delta(n)} e^{i n \theta}=O\left\{b(m)\left[d^{\prime}(m)\right]^{-1 / 2}\right\}
$$

where $u_{1}=n_{\mu+1}, v_{1}=n_{\mu+1}^{3 / 5}$. This shows that 


$$
R(m, \theta)=O\left\{\sum_{\nu=1}^{\mu} b\left(n_{\nu}\right) n_{\nu}^{2 / 5+\alpha}+\sum_{n=1}^{m} b(n) n^{2 \epsilon-1}+b(m)\left[d^{\prime}(m)\right]^{-1 / 2}\right\} .
$$

2. The order of $G(z)$. If the series

$$
\sum_{\nu=1}^{\infty} b\left(n_{v}\right)\left[d^{\prime}\left(n_{v}\right)\right]^{-1 / 2}
$$

converges uniformly in $\theta$, then the function $G(z)$ tends to a continuous function of $\theta$ as $\rho=|z| \rightarrow 1$. We shall now suppose that the series does not converge and investigate the order of $G(z)$ as $\rho \rightarrow 1$. We notice some properties of $n_{\nu}(\theta)$. First, since $d(x)$ is monotone, $n_{\nu}(\theta) \uparrow \infty$ as $\nu \rightarrow \infty$ and $n_{\nu}(\theta)$ is monotone decreasing as a function of $\theta$ on $(0,2 \pi)$. Also

$$
n_{\nu}(0)=n_{\nu+1}(2 \pi) \text {. }
$$

Finally, since

$$
d\left\{n_{\nu}(\theta)\right\}=2 \pi \nu-\theta
$$

we have

$$
d^{\prime}\left\{n_{\nu}(\theta)\right\} \frac{d}{d \nu} n_{\nu}(\theta)=2 \pi
$$

and, since $d^{\prime}(x)=O(1 / x)$, there must be a constant $c>0$ such that

$$
n_{\nu+1}(\theta)=n_{\nu}(\theta)+\int_{\nu}^{\nu+1} \frac{d}{d \mu} n_{\mu}(\theta) d \mu>n_{\nu}(\theta)+c n_{\nu}(\theta)=(1+c) n_{\nu}(\theta) .
$$

We let $h(x)=b(x)\left[d^{\prime}(x)\right]^{-1 / 2}$ and, if $n_{\mu}(0) \leqq x<n_{\mu+1}(0)$, we define

$$
M(x)=\sum_{\nu=2}^{\mu} \max _{n_{\nu-1}(0) \leqq y \leqq n_{\nu}(0)} h(y)+\max _{n_{\mu}(0) \leqq y \leqq x} h(y) .
$$

If $A$ is greater than 1 and $n_{\mu}(0) \leqq x<n_{\mu+1}(0), n_{\mu^{\prime}}(0) \leqq A x<n_{\mu^{\prime}+1}(0)$, then by (2.1) we have

$$
\mu^{\prime}-\mu<\log A[\log (1+c)]^{-1},
$$

and by (C), $h(y)=O\{h(x)\}, x \leqq y \leqq A x$, so that, if $n_{\mu}(0) \leqq x \leqq n_{\mu+1}(0)$, then

(2.2) $M(A x)=\sum_{\nu=2}^{\mu} \max _{n_{\nu-1}(0) \leqq y \leqq n_{y}(0)} h(y)+O\left\{\max _{n_{\mu}(0) \leqq y \leqq x} h(y)\right\}=O\{M(x)\}$.

We write

$$
|G(z)| \leqq 2 \pi^{1 / 2} \sum_{\nu=1}^{\infty} h\left(n_{\nu}\right) \rho^{n_{\nu}}=2 \pi^{1 / 2} \sum_{\nu=1}^{\infty} h\left(n_{\nu}\right) e^{y n_{\nu}}, \quad y=\log \rho,
$$


and define $\mu$ so that $n_{\mu}(\theta) \leqq-1 / y<n_{\mu+1}(\theta)$. Then

$$
\sum_{\nu=1}^{\infty} h\left(n_{\nu}\right) e^{y n_{\nu}}=\sum_{\nu=1}^{\mu} h\left(n_{\nu}\right) e^{\nu n_{\nu}}+\sum_{\nu=\mu+1}^{\infty} h\left(n_{\nu}\right) e^{\nu n_{\nu}}
$$

and, if $0 \leqq \theta<2 \pi$,

$$
\sum_{\nu=1}^{\mu} h\left(n_{v}\right) e^{y n_{\nu}}=O\left\{\sum_{\nu=1}^{\mu} h\left(n_{v}\right)\right\}=O\left\{M\left[n_{\mu}(\theta)\right]\right\} .
$$

By (C)

$$
\begin{aligned}
\sum_{\nu=\mu+1}^{\infty} h\left(n_{v}\right) e^{y n_{\nu}} & =h\left(-\frac{1}{y}\right) \sum_{\nu=\mu+1}^{\infty} h\left(n_{\nu}\right)\left[h\left(-\frac{1}{y}\right)\right]^{-1} e^{y n_{\nu}} \\
& =O\left\{h\left(-\frac{1}{y}\right) \sum_{\nu=\mu+1}^{\infty}\left(-n_{\nu} y\right)^{\log k} e^{n_{\nu} y}\right\} .
\end{aligned}
$$

Since $-n_{\nu} y>(1+c)^{\nu-\mu}\left(-n_{\mu} y\right)>(1+c)^{\nu-\mu-1}$, we may find an $A$ and an $m$ not depending on $y$ so that

$$
n_{\nu} y+\log k \log \left(-n_{\nu} y\right)<-A(\nu-\mu), \text { for }(\nu-\mu)>m .
$$

This shows that

$$
\begin{aligned}
\sum_{\nu=\mu+1}^{\infty} h\left(n_{\nu}\right) e^{\nu n_{\nu}} & =O\left\{h\left(-\frac{1}{y}\right) \sum_{\mu=\nu+1}^{\infty} \exp \left[n_{\nu} y+\log k \log \left(-n_{\nu} y\right)\right]\right\} \\
& =O\left\{h\left(-\frac{1}{y}\right)\right\} .
\end{aligned}
$$

Hence by (2.2)

$$
G(z)=O\left\{M\left(\frac{1}{1-\rho}\right)\right\} .
$$

We shall now use the additional hypothesis

$$
M(x)=O\{h(x)\}
$$

and we propose to prove that if (D) is satisfied then

$$
G(z)=\Omega\left\{h\left(\frac{1}{1-\rho}\right)\right\}=\Omega\left\{M\left(\frac{1}{1-\rho}\right)\right\} .
$$

The method is essentially that used by Hardy [1] for a similar problem. We take the series

$$
\sum_{v=1}^{\infty} n_{\nu}^{\alpha} h\left(n_{v}\right) e^{y n_{\nu}} e^{i F(v: \theta)}
$$


and let $y=-\alpha / n_{\mu}$. Then

$$
\left|\sum_{\nu=1}^{\infty} n_{\nu}^{\alpha} h\left(n_{\nu}\right) e^{y n_{\nu}} e^{i F(v ; \theta)}\right| \geqq n_{\mu}^{\alpha} h\left(n_{\nu}\right) e^{-\alpha}-\sum_{\nu=1}^{\mu-1} n_{\nu}^{\alpha} h\left(n_{\nu}\right) e^{\nu n_{\nu}}-\sum_{\nu=\mu+1}^{\infty} n_{\nu}^{\alpha} h\left(n_{\nu}\right) e^{y n_{\nu}} .
$$

By (D) we have $h\left(n_{\nu}\right)=O\left\{h\left(n_{\mu}\right)\right\}, \nu<\mu$, and hence there exists a $\lambda>0$ and independent of $\alpha, \nu$, and $\lambda$, such that

Therefore

$$
\left(\frac{n_{\nu}}{n_{\mu}}\right)^{\alpha} \frac{h\left(n_{\nu}\right)}{h\left(n_{\mu}\right)}<\lambda\left(\frac{n_{\nu}}{n_{\mu}}\right)^{\alpha}
$$

$$
\begin{aligned}
\sum_{\nu=1}^{\mu-1} n_{\nu}^{\alpha} h\left(n_{\nu}\right) \exp \left[-\alpha n_{\nu} / n_{\mu}\right] & <\lambda h\left(n_{\mu}\right) n_{\mu}^{\alpha} e^{-\alpha} \sum_{\nu=1}^{\mu-1}\left(\frac{n_{\nu}}{n_{\mu}}\right)^{\alpha} \exp \left[-\alpha\left(\frac{n_{\nu}}{n_{\mu}}-1\right)\right] \\
& =\lambda h\left(n_{\mu}\right) n_{\mu}^{\alpha} e^{-\alpha} \sum_{\nu=1}^{\mu-1} \exp \left[-\alpha\left(\frac{n_{\nu}}{n_{\mu}}-1-\log \frac{n_{\nu}}{n_{\mu}}\right)\right] .
\end{aligned}
$$

But, since $n_{\nu} / n_{\mu}<(1+c)^{-1}$, for $\nu<\mu$, there exists a $p>0$ such that

$$
\frac{n_{\nu}}{n_{\mu}}-1-\log \frac{n_{\nu}}{n_{\mu}}>p, \quad \text { for } \quad \nu<\mu,
$$

and, for $\mu-\nu>u, u$ depending only on $c$, there exists an $A$ so that

$$
\frac{n_{\nu}}{n_{\mu}}-1-\log \frac{n_{\nu}}{n_{\mu}}>A[\log (1+c)](\mu-\nu) .
$$

Therefore

$$
\begin{aligned}
& \sum_{\nu=1}^{\mu-1}\left(\frac{n_{\nu}}{n_{\mu}}\right)^{\alpha} \exp \left[-\alpha\left(\frac{n_{\nu}}{n_{\mu}}-1\right)\right]<\sum_{\nu=1}^{\mu-u-1} \exp [-\alpha A(\mu-\nu) \log (1+c)] \\
& \quad+\sum_{\nu=-\mu}^{\mu-1} e^{-\alpha p}<\sum_{\nu=1}^{\infty} \exp [-\alpha A \nu \log (1+c)]+u e^{-\alpha p}=o(1) \text { as } \alpha \rightarrow \infty .
\end{aligned}
$$

We have also by (C)

$$
\begin{aligned}
\sum_{\nu-\mu+1}^{\infty} n_{\nu}^{\alpha} & h\left(n_{\nu}\right) \exp \left(-\alpha n_{\nu} / n_{\mu}\right) \\
= & O\left\{n_{\mu}^{\alpha} h\left(n_{\mu}\right) e^{-\alpha} \sum_{\nu=\mu+1}^{\infty}\left(\frac{n_{\nu}}{n_{\mu}}\right)^{\alpha+\log k} \exp \left[-\alpha\left(\frac{n_{\nu}}{n_{\mu}}-1\right)\right]\right\} \\
= & O\left\{n_{\mu}^{\alpha} h\left(n_{\mu}\right) e^{-\alpha} \sum_{\nu=\mu+1}^{\infty}\left[-\alpha\left(\frac{n_{\nu}}{n_{\mu}}-1-\log \frac{n_{\nu}}{n_{\mu}}\right)+\log k \log \frac{n_{\nu}}{n_{\mu}}\right]\right\}
\end{aligned}
$$

and, since $n_{\nu} / n_{\mu}>(1+c)$ for $\nu>\mu$, there exists a $p>0$ for which, 


$$
\frac{n_{\nu}}{n_{\mu}}-1-\log \frac{n_{\nu}}{n_{\mu}}>p,
$$

and hence for $\alpha$ greater than a suitable $\alpha_{0}$, there is a $p_{0}>0$, such that

$$
\alpha\left(\frac{n_{\nu}}{n_{\mu}}-1-\log \frac{n_{\nu}}{n_{\mu}}\right)-\log k \log \frac{n_{\nu}}{n_{\mu}}>\alpha p_{0} .
$$

For $\nu-\mu>w$, $w$ independent of $\alpha$ for $\alpha>\alpha_{0}$,

$$
\alpha\left(\frac{n_{\nu}}{n_{\mu}}-1-\log \frac{n_{\nu}}{n_{\mu}}\right)+\log k \log \frac{n_{\nu}}{n_{\mu}}>\alpha[\log (1+c)](\nu-\mu) .
$$

Hence

$$
\begin{aligned}
\sum_{\nu=\mu+1}^{\infty} \exp \left[-\alpha\left(\frac{n_{\nu}}{n_{\mu}}\right.\right. & \left.\left.-1-\log \frac{n_{\nu}}{n_{\mu}}\right)-\log k \log \frac{n_{\nu}}{n_{\mu}}\right] \\
& <w e^{-\alpha p_{0}}+\sum_{\nu=1}^{\infty} \exp [-\alpha \nu \log (1+c)]=o(1) \text { as } \alpha \rightarrow \infty .
\end{aligned}
$$

Consequently we can find an $\alpha_{1}$ so that for $\alpha>\alpha_{1}$,

$$
\sum_{\nu=1}^{\mu-1} n_{\nu}^{\alpha} h\left(n_{\nu}\right) \exp \left(-\alpha n_{\nu} / n_{\mu}\right)+\sum_{\nu=\mu+1}^{\infty} n_{\nu}^{\alpha} h\left(n_{\nu}\right) \exp \left(-\alpha n_{\nu} / n_{\mu}\right)<\frac{1}{2} n_{\mu}^{\alpha} h\left(n_{\mu}\right) e^{-\alpha}
$$

uniformly in $\theta$ and $\mu$, and by (C), for $y=-\left(\alpha / n_{\mu}\right)$,

$$
\left|\frac{d^{\alpha}}{d \rho^{\alpha}} G(z)\right|>\frac{1}{2} \pi^{1 / 2} n_{\mu}^{\alpha} h\left(n_{\mu}\right) e^{-\alpha}>\omega\left(-\frac{1}{y}\right)^{\alpha} h\left(-\frac{1}{y}\right), \quad \omega \text { depends on } \alpha .
$$

We can easily see that

$$
\frac{d^{\alpha}}{d \rho^{\alpha}} G(z)=O\left\{\left(-\frac{1}{y}\right)^{\alpha} h\left(-\frac{1}{y}\right)\right\} .
$$

Therefore by a theorem of Hardy and Littlewood* we must have

$$
G(z)=\Omega\left\{h\left(\frac{1}{1-\rho}\right)\right\} .
$$

Therefore under condition (D) we have an exact characterization of the order of $G(z)$ as $|z| \rightarrow 1$.

3. The order of $R(z)$. By the above method we can easily show that

$$
\sum_{\nu=1}^{\infty} b\left(n_{\nu}\right) n_{\nu}^{2 / 5+\alpha} \rho{ }^{n_{\nu}}=O\left\{K_{1}\left(\frac{1}{1-\rho}\right)\right\}
$$

${ }^{*}$ Hardy and Littlewood [3]. This result follows from Theorem 8 on setting $\phi=\psi=h(-1 / y)$. 
where, if $n_{\mu}(0) \leqq x<n_{\mu+1}(0)$,

$$
K_{1}(x)=\sum_{\nu=2}^{\mu} \max _{n_{\nu-1}(0) \leqq y \leqq n_{\nu}(0)} b(y) y^{2 / 5+\alpha}+\max _{n_{\mu}(0) \leqq y<x} b(y) y^{2 / b+\alpha} .
$$

Also

$$
\sum_{\nu=1}^{\infty} b\left(n_{\nu}\right) n_{\nu}^{6 / 5+c} \rho{ }_{\rho}^{n \nu}=O\left\{K_{2}\left(\frac{1}{1-\rho}\right)\right\}
$$

where, if $n_{\mu}(0) \leqq x<n_{\mu+1}(0)$,

$$
K_{2}(x)=\sum_{\nu=2}^{\mu} \max _{n_{\nu-1}(0) \leqq y \leqq n_{\nu}(0)} b(y) y^{6 / 5+e}+\max _{n_{\mu}(0) \leqq y \leqq x} b(y) y^{6 / b+e}=O\left\{x^{4 / 5} K_{1}(x)\right\} .
$$

Similarly we can show that

$$
\sum_{n=1}^{\infty} b\left(n_{\nu}\right) n_{\nu}^{2 / 5+\alpha}{ }^{n_{\nu} / 2}=O\left\{K_{1}\left(\frac{2}{1-\rho}\right)\right\}=O\left\{K_{1}\left(\frac{1}{1-\rho}\right)\right\}
$$

and

$$
\sum_{\nu=1}^{\infty} b\left(n_{\nu}\right) n_{\nu}^{6 / 5+e} \rho^{n_{\nu} / 2}=O\left\{K_{2}\left(\frac{2}{1-\rho}\right)\right\}=O\left\{(1-\rho)^{-4 / 5} K_{1}\left(\frac{1}{1-\rho}\right)\right\} .
$$

Since $2 / 5+\alpha<\frac{1}{2}$, we have

$$
x^{2 / 5+\alpha}=o\left\{\left[d^{\prime}(x)\right]^{-1 / 2}\right\}
$$

and hence, if $M(x) \rightarrow \infty$ as $x \rightarrow \infty$,

$$
K_{1}(x)=o\{M(x)\} .
$$

Therefore, if $M(x) \rightarrow \infty$ as $x \rightarrow \infty$,

$$
R_{j}(z)=o\left\{M\left(\frac{1}{1-\rho}\right)\right\}, \quad j=1,2,3,4 .
$$

If $N \leqq-1 / y<N+1, y=\log \rho$, by (C)

$$
\sum_{n=N+1}^{\infty} b(n) n^{2 \epsilon-1} e^{y n}=O\left\{\sum_{j=0}^{\infty} \frac{b\left(2^{i} N\right)}{\left(2^{i} N\right)^{1-2 \epsilon}} \sum_{n=2^{i} N+1}^{2 j+1 N} e^{-n / N}\right\}
$$

and, since

$$
b\left(2^{i} N\right)\left(2^{i} N\right)^{2 \epsilon-1}=O\left\{2^{i \log k} b(N)\left(2^{i} N\right)^{2 \ell-1}\right\},
$$

we have 
Therefore

$$
\begin{aligned}
\sum_{n=N+1}^{\infty} b(n) n^{2 \epsilon-1} \rho^{n} & =O\left\{b(N) N^{2 \epsilon-1} \sum_{j=0}^{\infty} 2^{j(\log k+2 \epsilon-1)} e^{-2^{i}}\right\} \\
& =O\left\{b(N) N^{2 \epsilon-1}\right\} .
\end{aligned}
$$

$$
R_{5}(z)=O\left\{\sum_{n=1}^{[1 /(1-\rho)]} b(n) n^{2 \epsilon-1}\right\} .
$$

Similarly

$$
R_{6}(z)=O\left\{(1-\rho) \sum_{n=1}^{[1 /(1-\rho)]} b(n) n^{\epsilon}\right\}=O\left\{\sum_{n=1}^{[1 /(1-\rho)]} b(n) n^{\epsilon-1}\right\} .
$$

If the series

$$
\sum_{v=1}^{\infty} b\left(n_{v}\right)\left[d^{\prime}\left(n_{v}\right)\right]^{-1 / 2}
$$

converges uniformly in $\theta$, it is easily seen from the above estimates that the functions $R_{i}(z)$ tend to a continuous function of $\theta$ as $\rho \rightarrow 1$, for $i=1,2,3,4$. Moreover, since $d^{\prime}(x)=O(1 / x)$ we must have $b(x)=O\left(x^{-1 / 2}\right)$ so that

converges and

$$
\sum_{n=1}^{\infty} b(n) n^{2 \epsilon-1}
$$

$$
\sum_{n=1}^{\infty} b(n) n^{\epsilon} \rho^{n}=o\left\{(1-\rho)^{-1}\right\} .
$$

Therefore in this case $R(z)$ is continuous for $|z|=1$.

We now wish to give some estimate for

$$
\sum_{n=1}^{x} b(n) n^{2 \epsilon-1}
$$

and compare it if possible with $M(x)$. If $b(x)$ is increasing, by the monotonicity of $b(x)$

and, since by (A)

$$
\sum_{n=1}^{N} b(n) n^{2 \epsilon-1}=O\left\{b(N) N^{2 \epsilon}\right\}
$$

we see that

$$
b(N) N^{1 / 2}=O\{M(N)\}
$$

$$
R_{j}(z)=o\left\{M\left(\frac{1}{1-\rho}\right)\right\}, \quad j=5,6
$$


If $b(x)$ is not increasing the problem might have to be considered differently in separate cases. However if we make the reasonable assumption that

$$
\sum_{n=1}^{x} b(n) n^{2 \epsilon-1}=O\left\{b(x) x^{2 \epsilon+\gamma}\right\}, \quad 2 \epsilon+\gamma<\frac{1}{2},
$$

then we see that in this case

$$
R_{j}(z)=o\left\{M\left(\frac{1}{1-\rho}\right)\right\} .
$$

This assumption is always satisfied for functions of the logarithmic-exponential type which is the type of function used in applications of this method.

4. The modulus of continuity. If a function $f(z)$ analytic for $|z|<1$ is continuous for $|z|=1$, we define the modulus of continuity of $f(z)$ as the function

$$
\omega(f, h)=\underset{\left|\theta_{1}-\theta_{\mathbf{s}}\right| \leqq h}{\text { l.u.b. }}\left|f\left(e^{i \theta_{1}}\right)-f\left(e^{i \theta_{2}}\right)\right| .
$$

We wish to develop a method of finding the order of $\omega(f, h)$. Let us suppose that there is a function $g(x)$ such that $g(x) \uparrow \infty$ as $x \rightarrow 1$, then we shall show that if

then

$$
f^{\prime}(z)=O\{g(\rho)\}
$$

$$
\omega(f, h)=O\left\{\int_{1-h}^{1} g(x) d x\right\},
$$

provided the latter integral exists. The method used has been used by Hardy and Littlewood [4].

We have

$$
\left|f\left(e^{i \theta_{1}}\right)-f\left(e^{i \theta_{2}}\right)\right|=\lim _{\rho \rightarrow 1}\left|f\left(\rho e^{i \theta_{1}}\right)-f\left(\rho e^{i \theta_{2}}\right)\right|,
$$

so that we need only show that

$$
\left|f\left(\rho e^{i \theta_{1}}\right)-f\left(\rho e^{i \theta_{2}}\right)\right|=O\left\{\int_{1-h}^{1} g(x) d x\right\}
$$

uniformly in $\rho<1$ and $\left|\theta_{1}-\theta_{2}\right| \leqq h$. Since $f^{\prime}(z)$ is analytic for $|z|<1$,

$$
f\left(\rho e^{i \theta_{1}}\right)-f\left(\rho e^{i \theta_{2}}\right)=\int_{\rho e^{i \theta_{2}}}^{\rho e^{i \theta_{1}}} f^{\prime}(z) d z=\left\{\int_{1}+\int_{2}+\int_{3}\right\} f^{\prime}(z) d z,
$$

where $\int_{1}$ is the integral taken along the radius $\theta=\theta_{2}$ from $\rho$ to $\rho-h$, and $\int_{3}$ 
is the similar integral along $\theta=\theta_{1}$ from $\rho-h$ to $\rho$, while $\int_{2}$ is the integral along the $\operatorname{arc}|z|=\rho-h$ from $\theta_{2}$ to $\theta_{1}$. Then

$$
\begin{aligned}
\int_{2} f^{\prime}(z) d z & =i(\rho-h) \int_{\theta_{2}}^{\theta_{1}} e^{i \theta} f^{\prime}\left[(\rho-h) e^{i \theta}\right] d \theta \\
& =O\left\{\left|\theta_{1}-\theta_{2}\right| g(\rho-h)\right\}=O\{h g(1-h)\} .
\end{aligned}
$$

Since $g(x) \uparrow \infty$ as $x \rightarrow 1$,

Similarly

$$
\begin{aligned}
\int_{1} f^{\prime}(z) d z & =\int_{\rho}^{\rho-h} e^{i \theta_{2} f^{\prime}\left(x e^{i \theta_{2}}\right) d x} \\
& =O\left\{\int_{\rho-h}^{\rho} g(x) d x\right\} \\
& =O\left\{\int_{1-h}^{1} g(x) d x\right\} .
\end{aligned}
$$

$$
\int_{3} f^{\prime}(z) d z=O\left\{\int_{1-h}^{1} g(x) d x\right\} .
$$

Finally we notice that, since $g(x) \uparrow \infty$ as $x \rightarrow 1$, .

$$
h g(1-h) \leqq \int_{1-h}^{1} g(x) d x .
$$

Therefore

$$
\omega(f, h)=O\left\{\int_{1-h}^{1} g(x) d x\right\} .
$$

5. Applications. To illustrate the scope of this method we shall apply it in detail to a particular function which has already been considered and point out some extensions of the usual results which may be obtained by this method. The function which we shall consider is

$$
f_{\beta \gamma}(z)=\sum_{n=2}^{\infty} n^{-1 / 2}(\log n)^{-\gamma} \exp \left[i \int_{1}^{n}(\log x)^{\beta} d x\right] z^{n}, \quad 0 \leqq \beta \leqq 1 .
$$

This is similar to the function

$$
\sum_{n=2}^{\infty} n^{-1 / 2}(\log n)^{-\gamma} \exp \left[i n(\log n)^{\beta}\right] z^{n}
$$

considered by Ingham [6]. Ingham's function could also be handled by these methods but the details would be slightly more complicated. 
For the function under consideration

$$
d(x)=(\log x)^{\beta}, \quad b(x)=x^{-1 / 2}(\log x)^{-\gamma}
$$

and

$$
\left(\log \left[n_{\nu}(\theta)\right]\right)^{\beta}=2 \pi \nu-\theta, \quad n_{\nu}(\theta)=\exp \left[(2 \pi \nu-\theta)^{1 / \beta}\right] .
$$

Therefore

$$
\begin{gathered}
d^{\prime}(x)=\beta x^{-1}(\log x)^{\beta-1}=O\left(x^{-1}\right), \quad \text { since } \beta \leqq 1, \\
\frac{1}{d^{\prime}(x)}=\frac{1}{\beta} x(\log x)^{\beta-1}=O\left(x^{1+\epsilon}\right), \quad \text { for every } \epsilon,
\end{gathered}
$$$$
d^{\prime \prime}(x)=O\left(x^{-2}\right) \text {; }
$$$$
b^{\prime}(x)=-\frac{1}{2} x^{-3 / 2}(\log x)^{-\gamma}-\gamma x^{-3 / 2}(\log x)^{-\gamma-1}=O\left\{x^{-3 / 2}(\log x)^{-\gamma}\right\}
$$

$$
\begin{aligned}
& =O\left\{b(x) x^{-1}\right\} ; & \\
e^{2} d^{\prime}\left(x^{\prime}\right) & >d^{\prime}(x), & x \leqq x^{\prime} \leqq e x, \\
e^{2} b\left(x^{\prime}\right) & >b(x), & x \leqq x^{\prime} \leqq e x .
\end{aligned}
$$

Since $\eta=\delta=0$ and $\epsilon$ can be made less than $1 / 30$, we have

$$
\begin{aligned}
R(z)= & O\left\{(1-\rho) \sum_{\nu=2}^{\infty} n_{\nu}^{7 / 10} \rho^{n_{\nu}}+\sum_{\nu=2}^{\infty} n_{\nu}^{-1 / 20} \rho^{n_{\nu}}\right. \\
& \left.\quad+(1-\rho) \sum_{n=1}^{\infty} n^{-3 / 5} \rho^{n}+\sum_{n=1}^{\infty} n^{-6 / 5} \rho^{n}\right\} \\
= & O\left\{(1-\rho)(1-\rho)^{-7 / 10}+1+(1-\rho)(1-\rho)^{-2 / 5}+1\right\},
\end{aligned}
$$

so that $R(z)$ is continuous when $|z|=1$. The function $G(z)$ is continuous for $|z|=1$ if

$$
\sum_{\nu=2}^{\infty} n_{\nu}^{-1 / 2}\left(\log n_{\nu}\right)^{-\gamma} n_{\nu}^{1 / 2}\left(\log n_{\nu}\right)^{(1-\beta) / 2}=\sum_{\nu=2}^{\infty}(2 \pi \nu-\theta)^{-\gamma / \beta-1 / 2+1 /(2 \beta)}
$$

converges, and the necessary and sufficient condition that (5.1) converge is

$$
\frac{\gamma}{\beta}+\frac{1}{2}-\frac{1}{2 \beta}>1, \quad \text { or } \quad \gamma>\frac{1}{2}(\beta+1) .
$$

This corresponds to the result obtained by Ingham.

We shall now consider the modulus of continuity of $f_{\beta \gamma}(z)$. We notice that the function $b(x)=x^{1 / 2}(\log x)^{-\gamma}$ satisfies (B) and (C) so that the discussion of $\S \S 2,3$ applies to the function

$$
f_{\beta \gamma}^{\prime}(z)=\sum_{n=1}^{\infty} n^{1 / 2}(\log n)^{-\gamma} \exp \left\{i \int_{1}^{n}(\log x)^{\beta} d x\right\} z^{n} .
$$


Since $\beta \leqq 1$,

$$
\begin{aligned}
M(x) & =\sum_{n_{\nu \leqq x}} n_{\nu-1}\left(\log n_{\nu-1}\right)^{-\gamma-\beta / 2+1 / 2}+x(\log x)^{-\gamma-\beta / 2+1 / 2} \\
& =O\left\{x(\log x)^{-\gamma-\beta / 2+1 / 2}\right\}
\end{aligned}
$$

so that

$$
f_{\beta \gamma}^{\prime}(z)=O\left\{(1-\rho)^{-1}\left[\log \left(\frac{1}{1-\rho}\right)\right]^{-\gamma-\beta / 2+1 / 2}\right\}
$$

But

$$
\begin{gathered}
\int_{1-h}^{1}(1-\rho)^{-1}\left[\log \left(\frac{1}{1-\rho}\right)\right]^{-\gamma-\beta / 2+1 / 2} d \rho=\int_{0}^{h} x^{-1}\left(\log \frac{1}{x}\right)^{-\gamma-\beta / 2+1 / 2} d x \\
=\int_{1 / h}^{\infty} x^{-1}(\log x)^{-\gamma-\beta / 2+1 / 2} d x=\frac{-1}{\gamma+\beta / 2-3 / 2}\left(\log \frac{1}{h}\right)^{-\gamma-\beta / 2+3 / 2}, \\
\text { if } \gamma>\frac{3}{2}-\frac{\beta}{2}
\end{gathered}
$$

and hence

$$
\omega\left(f_{\beta \gamma}, h\right)=O\left\{\left(\log \frac{1}{h}\right)^{-\gamma-\beta / 2+3 / 2}\right\}, \quad \text { if } \quad \gamma>\frac{3}{2}-\frac{\beta}{2} .
$$

This result could not be obtained by the other methods to which we have referred.

6. Further applications. If

$$
\sum_{n=1}^{\infty} b^{2}(n)=\infty,
$$

we know that the function

$$
f(z)=\sum_{n=1}^{\infty} b(n) e^{i \Delta(n)} z^{n}
$$

can not tend to a continuous or even a bounded function as $\rho=|z| \rightarrow 1$. We wish now to study the behavior of $f\left(\rho e^{i \theta}\right)$ for a particular choice of $d(x)$. We assume that $b(x)$ satisfies (B) with $\delta<1 / 10-\epsilon$, and (C) and,

$$
\int_{1}^{x} b^{2}(t) d t=O\left\{x^{1+\epsilon} b^{2}(x)\right\}, \quad \epsilon<\frac{1}{30} .
$$

We define

$$
d(x)=\log \left[\int_{1}^{x} b^{2}(t) d t\right]
$$


Then by hypothesis $d(x) \uparrow \infty$, as $x \rightarrow \infty$, and

$$
\begin{aligned}
d^{\prime}(x) & =\frac{b^{2}(x)}{\int_{1}^{x} b^{2}(t) d t}=O\left(x^{-1}\right), \\
d^{\prime \prime}(x) & =O\left(x^{\delta-2}\right), \quad \delta<\frac{1}{10}-\epsilon, \\
\frac{1}{d^{\prime}(x)} & =O\left(x^{1+\epsilon}\right), \quad \epsilon<\frac{1}{30},
\end{aligned}
$$

so that the function $d(x)$ so defined satisfies (A). It is also clear that since $b(x)$ satisfies (C), $d(x)$ will also satisfy (C). We have

$$
b(x)\left[d^{\prime}(x)\right]^{-1 / 2}=\left(\int_{1}^{x} b^{2}(t) d t\right)^{1 / 2} \uparrow \infty \quad \text { as } \quad x \rightarrow \infty
$$

and

$$
b\left(n_{v}\right)\left[d^{\prime}\left(n_{v}\right)\right]^{-1 / 2}=e^{\pi v-\theta / 2} .
$$

Therefore

$$
\begin{aligned}
M(x) & =\sum_{n_{\nu}(0) \leqq x} b\left(n_{\nu}(0)\right)\left[d^{\prime}\left(n_{\nu}(0)\right)\right]^{-1 / 2}+b(x)\left[d^{\prime}(x)\right]^{-1 / 2} \\
& =\sum_{n_{\nu}(0) \leqq x} e^{\pi \nu}+b(x)\left[d^{\prime}(x)\right]^{-1 / 2}=O\left\{b(x)\left[d^{\prime}(x)\right]^{-1 / 2}\right\}
\end{aligned}
$$

and condition (D) is satisfied. By the argument of $\$ 3$

$$
R(z)=o\left\{M\left(\frac{1}{1-\rho}\right)\right\}+o\left\{\sum_{1}^{[1 /(1-\rho)]} b(n) n^{2 \epsilon-1}\right\}
$$

and

$$
\begin{aligned}
\sum_{1}^{N} b(n) n^{2 e-1} & \leqq\left[\sum_{1}^{N} n^{-1 / 2} b^{2}(n)\right]^{1 / 2}\left[\sum_{1}^{N} n^{4 \epsilon-3 / 2}\right]^{1 / 2} \\
& =o\{M(N)\}
\end{aligned}
$$

so that

$$
R(z)=o\left\{M\left(\frac{1}{1-\rho}\right)\right\}
$$

Therefore by the argument of $\$ 2$,

$$
f(z)=O\left\{M\left(\frac{1}{1-\rho}\right)\right\}=O\left\{\left(\int_{1}^{1 /(1-\rho)} b^{2}(x) d x\right)^{1 / 2}\right\}
$$


and

$$
f(z)=\Omega\left\{M\left(\frac{1}{1-\rho}\right)\right\} .
$$

This answers the question mentioned in the introduction about the possibility of choosing $d(x)$ so that $f(z)$ have the same order as $|z| \rightarrow 1$ for every $\theta$.

We might apply these considerations to the case where $b(x)=x^{-1 / 2}$. We can readily obtain the result for this case that if

$$
d(x)=\log \left[\int_{1}^{x} \frac{d t}{t}\right]=\log \log x
$$

then

$$
f(z)=\sum_{n=3}^{\infty} n^{-1 / 2} \exp [i \Delta(n)] z^{n}=O\left\{\left[\log \left(\frac{1}{1-\rho}\right)\right]^{1 / 2}\right\}
$$

and

$$
f(z)=\Omega\left\{\left[\log \left(\frac{1}{1-\rho}\right)\right]^{1 / 2}\right\} .
$$

We might compare this with the results for the function

$$
f_{0}(z)=\sum_{n=2}^{\infty} n^{-1 / 2} \exp [\text { in } \log n] z^{n}
$$

obtained by Hardy and Littlewood which are mentioned in the introduction ((1) and (2)).

\section{REFERENCES}

1. Hardy, these Transactions, vol. 17 (1916), pp. 301-325.

2. Hardy and Littlewood, Proceedings of the National Academy of Sciences, vol. 2 (1916), pp. 583-586.

3. Hardy and Littlewood, Proceedings of the London Mathematical Society, (2), vol. 11 (1912), pp. 411-478.

4. Hardy and Littlewood, Mathematische Zeitschrift, vol. 28 (1928), pp. 512-634.

5. Hille, Journal of the London Mathematical Society, vol. 4 (1929), pp. 176-182.

6. Ingham, Annals of Mathematics, (2), vol. 31 (1930), pp. 241-250.

7. Paley, Proceedings of the London Mathematical Society, (2), vol. 31 (1930), pp. 301-328.

8. Wilton, Journal of the London Mathematical Society, vol. 9 (1934), pp. 194-201, 247-256.

NORTHWESTERN UNIVERSITY,

Evanston, Ill. 